\title{
CASE OF INTEREST
}

\author{
Crohn's Disease
}

\begin{abstract}
Aetiology
THE aetiology of Crohn's disease still remains unexplained, ileo-caecal tuberculosis, sarcoid, other granulomata and numbers of other causes have been postulated but so far nothing positive has emerged to explain this mysterious disease which is far from being a rarity, for between July 1967 and February 1970 we saw in the British Military Hospital, Iserlohn no less than 11 cases, six proved by laparotomy and five by $\mathrm{X}$-rays or biopsy, and others were suspected.

As has been mentioned, exhaustive investigations and enquiry have so far failed to identify an aetiological factor, but it is submitted that the clinical stage of Crohn's disease might be the end result of a disorder of function consequent on an autotonomic dysrhythmia of the intestinal tract, and in particular of the colon, caecum and the adjacent terminal ileum.
\end{abstract}

The motility of the intestinal tract and its blood supply have been amply demonstrated to be profoundly influenced by changes of mood and emotion, and the mucous membrane of the stomach has been observed to reflect faithfully, irascibility, depression and excitement. Whilst colonic pain and mucous diarrhoea have long been noted to be symptoms of stress in certain people, particularly those thin pale dark haired individuals of both sexes, who tend to nurse their ills and grievances rather than give vent to their feelings in an explosion of temper.

Mucous colitis or spastic colon has for years been recognised as an intestinal neurosis and one must ponder what relation this has to Crohn's disease of the colon and terminal ileum and in what way alimentary dysrhythmia of these regions can progress to this organic disease.

For the great majority of cases involved the ileo-caecal region and colon, and when the upper small bowel is involved, which is not common, it usually exists with distal small bowel disease, so perhaps it is not unreasonable to assume that its aetiology may lie in factors which are found in the colon and ileo-caecal region to a greater degree than elsewhere in the intestinal tract.

To begin with, the colon, although of comparative short length, is in far longer contact with intestinal contents, which are rather more solid, than is to be found in other segments of bowel and in it a condition of relative stasis exists. Faecal material therefore provides a prolonged stimulus to the gut wall and this will be particularly marked in those individuals who by reason of temperament and personality are the subjects of colonic dysrhythmia, manifested by sustained tonic irregular contractions and reversed peristalsis combined with which may be a tonic ileo-caecal valve, for it is a common clinical finding to palpate, particularly in cases of spastic colon a tender iliac colon and caecum.

It is not inconceivable therefore that under these curcumstances a peristaltic wave in the terminal ileum may find the ileo-caecal valve closed firmly against it, and as a consequence it is not difficult to visualise spasm of tonic type developing in this segment of bowel. Conversely, reverse colonic peristalsis may permit regurgitation of colonic 
contents into the distal ileum where the reaction would again be that of tonic spasm as a reflex against foreign matter.

\section{Pathogenesis}

The question now to be considered is how a state of dysfunction can progress to clinical Crohn's disease, for it is indeed a far cry from a dysrhythmia to a severely pathological state where bowel and omentum may be grossly thickened, fistulae, internal and external may form, and abscesses, ascites and considerable lymphadenopathy develop with consequent serious danger to the patient's health.

It is suggested that the explanation may be that prolonged spasm and dysfunction which cause a rise in the intra luminal pressure of the colon and terminal ileum results in a transudation of plasma protein from blood vessels in the intestinal wall into the tissue spaces where normally only a trace of protein exists.

This may cause a local auto-immune reaction rather similar to the role of thyroglobulin in Hashimoto's disease, and may well explain the curious skip lesions as they are called which are found at intervals along the length of the colon separated by normal tissue, and which are very characteristic of Crohn's disease.

Indeed Crohn's disease has many of the features of an auto-immune illness with the frequent responses of temperature, E.S.R. and other symptoms to the administration of ACTH and Steroids.

Colonel H. FOSTER 